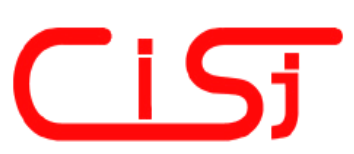

\title{
MULTI-CHANNEL MAGNETOCARDIOGRAPH: CONTROL AND SOFTWARE
}

\author{
Igor Voytovych ${ }^{1)}$, Myhailo Primin ${ }^{2)}$, Valery Vasyliev ${ }^{3)}$, Pavlo Sutkovyy ${ }^{3)}$, Mykola \\ Budnyk ${ }^{3)}$, Igor Nedayvoda ${ }^{4)}$, Anatoly Rusanov ${ }^{4}$, Tetyana Ryzhenko ${ }^{4)}$
}

Glushkov Institute for Cybernetics, prospect Academika Glushkova 40, 03680 Kyiv-187, Ukraine Phone: 380-44-2660128, 266-1267, Fax: 380-44-2663348, E-mail: d220@public.icyb.kiev.ua

1) $\mathrm{PhD}$, Prof., Corr. Member of the NAS of the Ukraine

2) $\mathrm{PhD}$, (contact author)

3) Candidates of Sciences

4) Researchers

\begin{abstract}
Purpose of the work was to present algorithms and software developed for working with multi-channel magneto-cardiograph. Such software is intended for control of operation and computer processing of magnetocardiographic (MCG) data obtained from the human heart. Magnetocardiograph is controlled as "virtual device" from PC mouse/keyboard, so as manually from electronic unit and all that through the control microprocessors embedded into hardware units. Software processing is performed both in on-line mode during process of data acquisition and in off-line manner during post-processing. Software allows preliminary processing, reconstruction and analysis of magnetic maps, and also inverse problem solution. Package is intended both for scientific studying of the heart electric activity and studying of MCG informative indexes for clinical diagnostic of cardiology diseases. Above software is planned to use at Strazhesko Institute of Cardiology (Kyiv) within framework of project supported by Science and Technology Center in Ukraine (STCU).
\end{abstract}

\section{INTRODUCTION}

Superconducting Quantum Interference Detectors (SQU-IDs) are characterized by the sensitivity of $(10 \div 15)^{-15} \mathrm{~T} / \sqrt{\mathrm{Hz}}$ which allows to register super-low fields connec-ted with the biological activity of living organisms. Magnetic fields are almost not disturbed by biological tissues, measurement is contact-free and passive. That is why, that SQUID-based measurements give a new direction in the study of biological objects.

Largest magnetic signal from the human body is generated by the heart. Due to above advantages, magnetocardiography is capable to give such information about heart, which cannot be obtained by other methods. Realization of measurements with the help of supersensitive SQUID sensors is a difficult problem. It is connected with solving a number of tasks concerning noise-immunity, filtration, multichannel data acquisition, signal processing, their representation, interpretation, etc.

We have created a multi-channel magnetocardiograph which operation is described in [1]. Signal processing is performed by specially designed software. Control and adjustment of device are performed by microprocessors embedded into electronics. Partially these materials were early published in [2-8].

\section{MICROPROCESSOR CONTROL SYSTEM}

Microprocessor control system (MCS) is intended in to control and adjust all channels of magnetometer. It is performed by 4 microprocessor embedded into electronic units. This system allows establishing modes of operation, indicates magnetometer status and provides technical service of the magnetometer. MCS together with computer software provides automatic control, releases operator from monotonous works and reduces time needed to put the magnetometer into operation, increases reliability of measurements.

Flowchart of the MCS is shown on Fig. 1 and following parameters are controlled: $\mathrm{I}_{0}, \mathrm{~F}_{0}, \mathrm{U}_{0}$, Gain, FeedBack, Reset, Heater, HelM. Gain is amplification of controlled amplifiers of magnetocardiography (MCG) channels with digital 
gain values $(=1,2,4,8)$. Other control parameters are described at Fig.1. In addition, there is possibility to plot transfer characteristics of the SQUID of all 7 channels.

System operation can be briefly described in such a way. The system includes 4 microprocessor modules: MP7, SW7, SW8, and RC7. Scheme of module links with each other and PC is shown on Fig.1. The modules have the following tasks: SW8 controls the Probe electronics of reference magnetometer, module SW7 controls the Probe electronics of 4 MCG channels and transmits commands for SW8 via itself. Modules SW7 and SW8 are installed in Intermediate modules of MCG and reference channels accordingly. Module MP7 is the major control module installed in Control Unit; it controls the electronic components in Control Unit and connects another modules and PC. Module RC7 locates in Technical control panel (TCP) used for system adjusting.

Information interchange protocol of modules between each other and the PC is made by standard
RS232. Inter-face parameters are: speed 9600 baud; no pair control, parcel length -8 bit, 1 stop bit. There are input devices, indicators and execution devices in the system. Input devices are the keys, by pressing which an operator inputs commands to change the state of device. Indication elements are light or sound indicators informing the operator about the device status. Executing elements are the electronic elements (switches, potentiometers etc.), changing of those positions result in changes of the device state.

Commands to change the state of the magnetometer are transmitted also from the PC. According to format and the mode, there are three types of commands:

1.Commands without a parameter bring an execution device into some fixed state (enabling or disabling). Command code selects execution device and its state, and is the byte, which came to the module through the channel RS232. After command executing (i.e. switching the device), the module returns the echo.

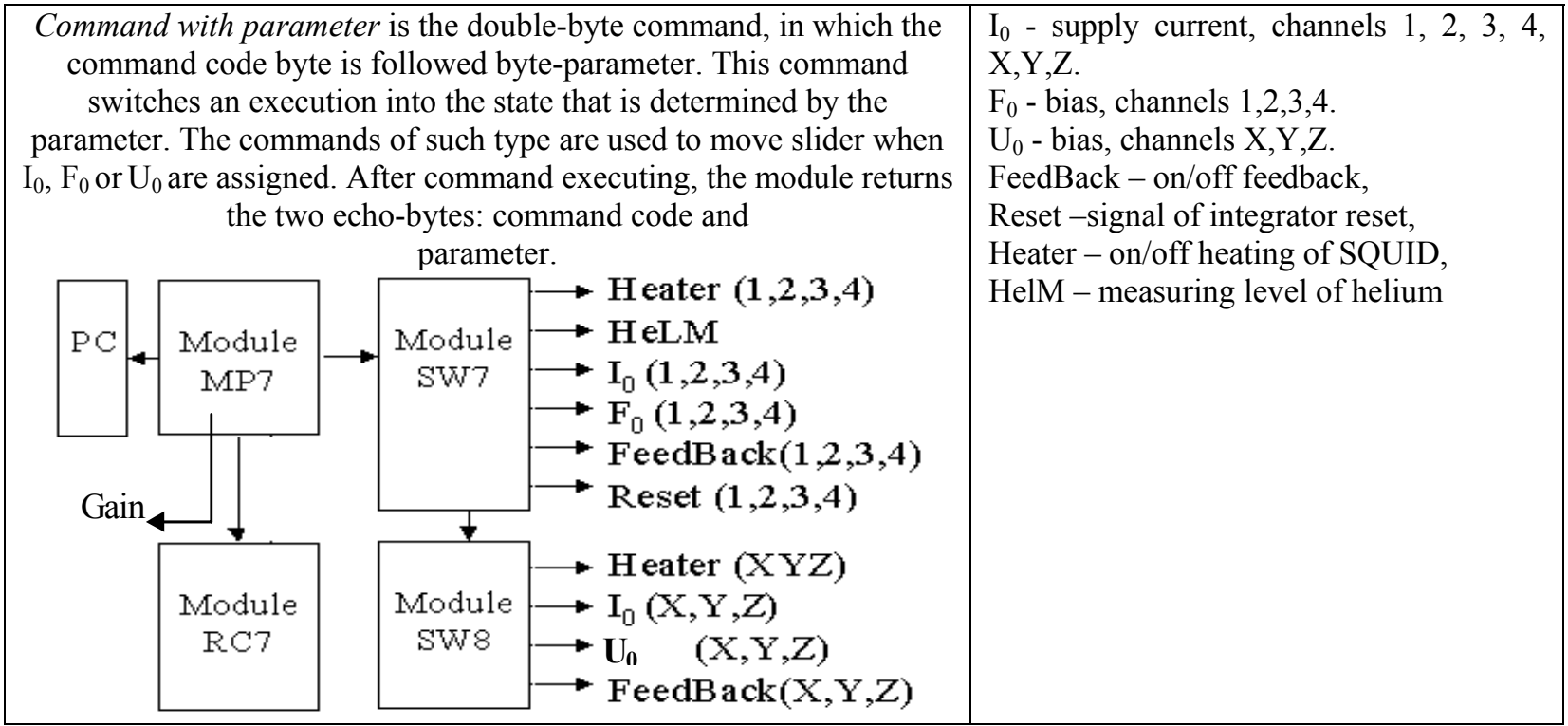

Fig. 1 - Flowchart of module connections for the microprocessor control system.

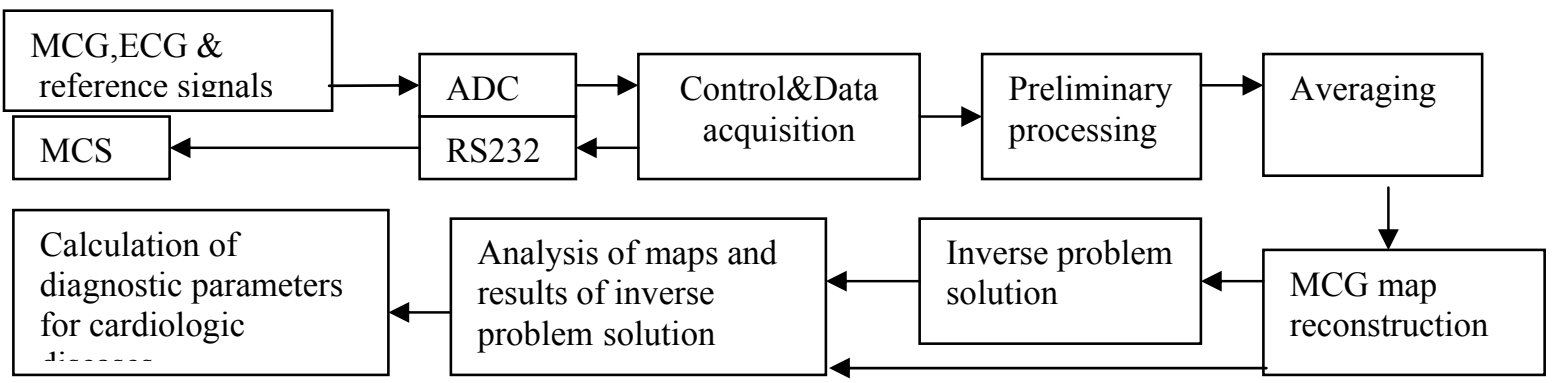

Fig.2 - Structure of the magnetocardiographic software.

2.Command asking parameter value is the single byte command. After obtaining the command code, module delivers the parameter value byte. 
In response to the command the module delivers 200 bytes.

\section{SOFTWARE PACKAGES}

Magnetocardiographic software consists of two separate packages. The first is used for input and preprocessing of MCG data. The output of this package is a binary file with $36 \mathrm{MCG}$ and 1 ECG averaged P-QRS-T complexes. The second package carries out advanced mathematical signal processing with the purpose of medical interpretation. Software structure is shown at Fig.2, where $1^{\text {st }}$ and $2^{\text {nd }}$ lines of boxes reflect $1^{\text {st }}$ and $2^{\text {nd }}$ packages, respectively.

\subsection{PACKAGE FOR CONTROL, DATA ACQUISITION, AND PRELIMINARY PROCESSING}

This package performs following functions:

- input of raw data to computer;

- controlling of the control unit;

- storing of data and patient's information in database;

- morphological analysis of ECG ;

- digital filtering of MCG;

- adaptive noise compensation (ANC) with the reference channels data;

- averaging of MCG P-QRS-T complexes.

Raw MCG signals should be preliminary processed because of very high level of magnetic noise in an unshielded room. The pre-processing procedures suppress noise and increase SNR up to thousands times.

The first step of preprocessing is the morphological analysis of ECG signals. It includes identification of QRS complexes, their classification (clustering) and determination of the base point and boundaries of every complex. Prior to the analysis, the low-pass filtration (LPF) $(<40$ $\mathrm{Hz})$ and high-pass one (HPF) $(>0,05 \mathrm{~Hz})$ are performed for bad quality ECG records. Automatic iterative procedures are applied to identify and group QRS complexes of different types (normal sinus rhythm, extra systole, etc). Results of ECG morphological analysis are used for averaging of correlated in time MCG complexes and to synchronize MCG records for different spatial positions.

For suppressing of external correlated noise in MCG data software uses data of the 3-channel reference vector magnetometer (RVM), which output signals are adaptively extracted from the MCG signals. The traditional digital noise filtering includes 3 steps:
1) LPF with the cutoff of $50 \mathrm{~Hz}$ to reject power line $50 \mathrm{~Hz}$ signal and its higher harmonics.

2) Narrow-band filtering to decrease and power lower sub-harmonics ( $25 \mathrm{~Hz}, 16.6 \mathrm{~Hz}$, etc) or any other narrow- band noise in the frequency range of MCG signal.

3) HPF with the cutoff of $0.5 \mathrm{~Hz}$ to decrease LF noise and baseline deviation.

But simple narrow- band filtering often essentially distorts the useful MCG signal. So instead of or additionally to third step the adaptive mixing of MCG and RVM channels data is used. The algorithm identifies optimum ratios between signals of MCG and RVM channels and executes adaptive subtraction of the filtered RVM data from the MCG ones.

The averaging algorithm is designed in order to improve SNR and synchronize MCG signals recorded at different spatial positions. The algorithm accumulates measured signal under synchronizing periodic sequence - ECG. Averaging procedure is executed in automatic mode. During automatic mode some the "worst" complexes (with jumps, spikes, short-pulses, etc) are automatically rejected from averaging. Any complexes may be manually rejected by operator to increase the averaged signal's quality.

\subsection{PACKAGE FOR ADVANCED DATA PROCESSING}

This package performs following tasks:

- 2D interpolation of 36 curves into area 20X20 $\mathrm{cm}$;

- selecting of cardiocycle's intervals;

- reconstruction of set of MCG maps;

- morphological analysis of MCG curves;

- quantitative analysis of MCG maps;

- inverse problem solution within dipole approach;

- inverse problem solution as current density plane;

- calculation of various dipole and currents' characteristics;

- finding of diagnostic parameters for different heart diseases.

Magnetic field mapping consists in splineinterpolation of the 36 measured nodes 2-D function at the points that are not the nodes and determination of isolines of magnetic field. The sequence of such maps displays a spatial structure and changing of magnetic field in any interval of P-QRS-T complex.

Analysis of MCG data for normal and ill patients may be more exact under consideration of magnetic field sources' parameters and characteristics. We have developed two models of 
sources, evaluated their parameters and built the algorithms for localization of zones of electric activity at different intervals of cardiocycle.. The $1^{\text {st }}$ model is the single magnetic dipole. And the $2^{\text {nd }}$ model is a plane layer with $2 \mathrm{D}$ current distribution.

To define the parameters of both models, the inverse problem must be solved. The original twostage analytical approach has been developed. The idea of this approach is based on properties of spatial derivatives tensor of the magnetic induction vector into a homogeneous, nonmagnetic, and non-conducting medium. Within $1^{\text {st }}$ stage, effective magnetic dipole is localized. At the $2^{\text {nd }}$ stage, within a plane, crossing a heart on a dipole distance, the distribution of current density is determined by means of double Fourier transformation [2].

During preliminary testing, data acquisition of MCG map from healthy volunteer has been made. It was shown that 4-channel magnetocardiograph with ANC allows registering MCG without magnetic shielding with quality level necessary for medical diagnosing.

\section{CONCLUSIONS AND ACKNOWLEDGMENTS}

Electronic control system and software for 7 (4 measurement and 3 reference)-channel magnetocardiograph were developed and preliminary tested. The software consists of two packages, first of them performs control, data acquisition, preliminary processing, and averaging. Second package provides magnetic map reconstruction, inverse problem solution, and analysis of results for the cardiology diagnostics. Algorithms of this advanced acquisition and processing software accumulates experience based both on earlier single-channel [2-6] and multichannel devices [7-8].

This work has been performed with the financial support from Science and Technology Center in Ukraine (STCU) within Grant No.2187.

\section{REFERENCES}

[1] I. Voytovych, M. Budnyk, Yu Minov. First Ukrainian multi-channel magnetocardiograph: assembling and testing, in this issue.

[2]M. Primin, V. Gumenyuk - Sychevsky, I. Nedayvoda. Methods and alhorithms of magnetic source localization, Naukova Dumka, Kyiv, 1992-92 c.

[3] N. Budnik, A. Gapelyuk, Yu Minov. Human biomagnetic signal measurement for diagnosing some diseases. Proc. 13 IMEKO World
Congress.-Torino (Italy), 1994, Vol.3 - p.25092514.

[4] I. Voytovych, Superconductive electronics and biomagnetism, Scientific issue. - Kyiv: Institute for Cybernetics, 1994.

[5] I. Voytovych, M. Budnyk, Yu Minov. Computer-Aided Biomagnetic Investigation Systems, USIM, 1995, - № 3, c. 31-46.

[6] M. Budnyk, I. Voytovych, V. Kozlovsky. Diagnostic criteria for chronic ishemic heart disease based on registry and analyses of the magnetocar-diograms, Preprint 2002-2, Institute for Cybernetics, Kyiv (Ukraine), 2002

[7] M. Primin, I. Nedayvoda, V. Vasylyev. Algorithm of axial gradiometers additional balan-cing in the not uniform magnetic field, BIOMAG' 2002, H. Nowak, J. Haueisen, F. Giessler, R. Huonker, eds. - VDE Verlag: Berlin-Offenbach, 2002. - p. 952-954.

[8] V. Vasylyev, M. Primin, I. Nedayvoda. Use of the reference magnetometric channels for noise suppression in biomagnetic investigations, Ibid. - p. 1036-1038.

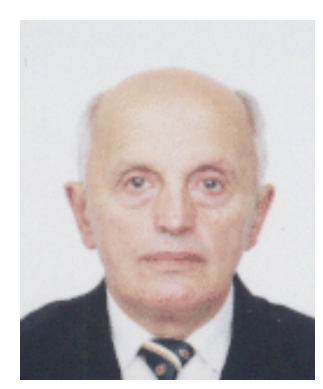

Igor Voytovych - Doctor of Technical Sciences, Professor, Cor. Member of National Academy of Sciences of Ukraine. He is expert in the field of superconductive electronics, microelectronics, computer engineering and instrumentmaking. He has over 200 works, including 60 inventions and monograph. He was born at Vinnytsya region in 1932. In 1956 after Lviv Technical University (TU) "Politechnika" he received diploma in radioengineering. From 1980 he defended PhD thesis and now is head of Cryoelectronic Department. During 2001-2004 he is manager of Scientific and Technology Center in Ukraine (STCU) project 2187 (granted by EC and Canada) resulting in magnetocardiographic (MCG) system developed and installed at cardiology clinic.

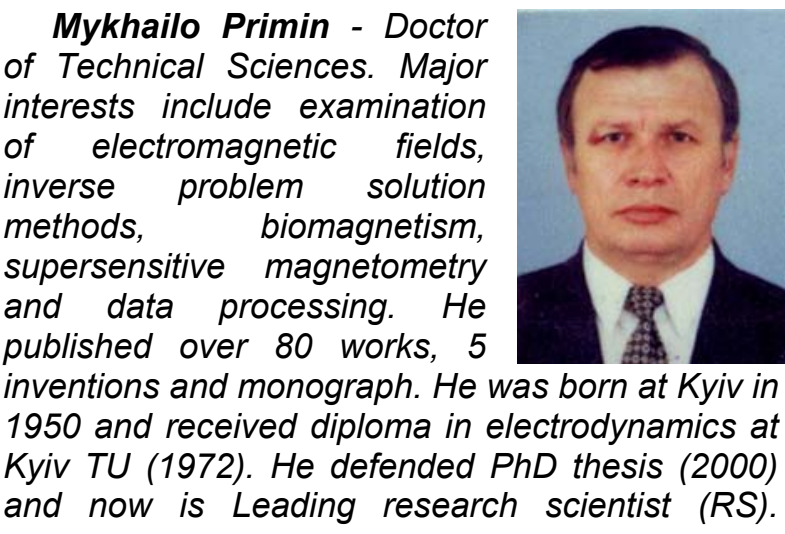


During last decade he took part in: creating of software for 10-channel MCG system of (Cryoton Ltd, Moscow); processing of data from FranzVolhard-Klinik (Berlin), development of software for 7-channel system (2002-2003, Essen, Germany) and software development within STCU project 2187.

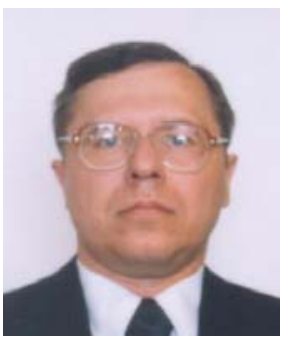

Mykola Budnyk -
Candidate of Engineering Sciences. He has over 100 works including 4 patents. Activity is focused on the studying of Superconducting Quantum Interference Devices (SQUIDs) and their applications, firstly magnetometers. Last years he interested in design of computer-aided systems for measure of biomagnetic fields and implementation of MCG technology into clinical practice. He was born in Sumy region at 1962. In 1984 after Kyiv National University (KNU) he received diploma in radiophysics. He defended Candidate thesis (1997), Senior RS 1998, and finished doctor fellowship (2002). He took part in creating of MCG devices in Germany (1999-2001, Essen). From 2002 he is co-manager of Biomagnetic Laboratory (BL) at Strazhesko Institute of Cardiology at Kyiv.

\section{Pavlo Sutkovyi -} Candidate of Technical Sciences, expert in the lowtemperature physics, cryoelectronics, radioelectronics automation $\mathrm{He}$ has 70 works and 5 inventions. He was born at Kyiv in 1957 and

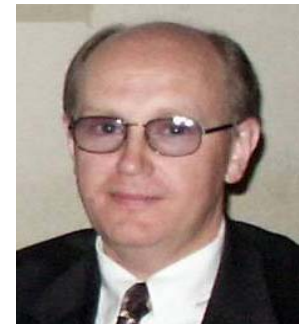
received diploma in radio-physics at KNU (1980). After defence of Candidate thesis he is Senior RS (1997). During 1999-2004 he worked at Essen (Germany) and took part in creating of 7-channel MCG systems and their installation into clinics. Also he collaborated with Jenasensoric (Jena, Germany) in development of microprocessor systems for multisensors. During 2001-2004 he developed electronics for MCG system within project 2187.

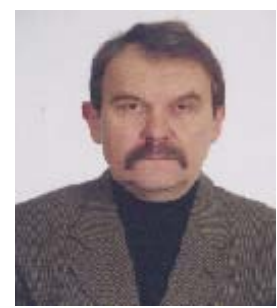

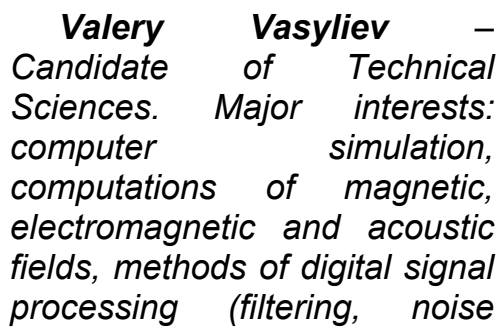
suppressing, frequency analysis, averaging, etc.). He is author of more than 50 works. He was born at Kyiv region in 1950 and received diploma in cybernetics of

electrical systems at Kyiv TU (1973). He defended Candidate thesis (1982) and now is Senior RS. During 2001-2004 he developed software for MCG system within project 2187.

Igor Nedayvoda - research fellow. He was born in 1962. In 1985 after Kyiv TU he received diploma in physics. $\mathrm{He}$ is co-author of monograph, published 40 works, and has 5 inventions. Main interest is data processing of magnetometric information, firstly from the human heart, computer databases, programming technology, and advanced numerical methods. During 2001-2004 he developed software for MCG system within project 2187.

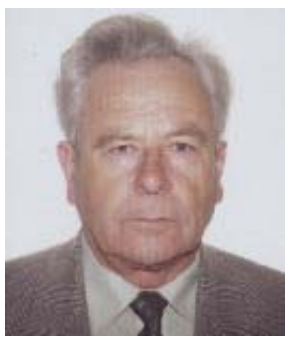

Anatoly Rusanov leading engineer. He was born at 1940 in Zaporizhzhya region. In 1965 after Lviv TU he received diploma in computer engineering He has 3 publications. Expert in the field of electronics and microprocessor-based apparatus. Last time he developed microprocessor-based control for MCG system within project 2187.

Tetyana Ryzhenko - She was born at Kyiv in 1978. In 2002 after Kyiv TU he received diploma in physical and biomedical engineering. She has 3 publications and now is Junior RS. Scientific interests include: implementation of MCG method into clinical practice, computer data processing of medical data including statistical, image analysis and pattern recognition approaches. 